\title{
Evaluación de modelos de diseño instruccional: una revisión de literatura
}

Ana Cristina Umaña Mata

Vicerrectoría Académica

Universidad Estatal a Distancia, Costa Rica

acumanam@uned.ac.cr

\section{Resumen}

En este artículo se presenta la reseña de literatura, efectuada en relación con el tema de la evaluación de modelos de diseño instruccional. El trabajo surge previo a la realización de una investigación, cuyo objetivo fue "evaluar el modelo de diseño de cursos de la UNED, con el fin de identificar los componentes que generan retraso en el proceso de producción de materiales instruccionales y proponer modificaciones al modelo" (Umaña, 2012, p. 6).

En la investigación bibliográfica realizada, se concluye que la evaluación de los modelos es un interés reciente, dado que, generalmente han sido validados en su puesta en práctica. Sin embargo, posterior a su implementación, el componente evaluativo es dejado de lado; lo cual limita las posibilidades de realizar mejoras y cambios, antes de que el modelo entre en un proceso de caducidad.

Se persigue, por tanto, un acercamiento teórico en relación con el tema y la discusión de aspectos fundamentales a considerar en la actualidad; teniendo en cuenta el creciente auge de los procesos de autoevaluación y acreditación educativa en las instituciones de educación superior.

Palabras clave: diseño instruccional, evaluación educativa, modelos de evaluación educativa, educación a distancia, materiales didácticos.

\section{Abstract \\ Evaluation models of instructional design: literature review}

This article presents the literature review conducted in relation to the subject of the evaluation of instructional design models. The work arises prior to the completion of an investigation whose objective was "to assess the design model of college courses, in order to identify the components that generate delay in the production process of instructional materials and propose modifications to the model" (Umaña, 2012, p. 6).

In the research literature is that the evaluation of the models is a recent interest since generally these have been well validated in its implementation. However, after its implementation, the evaluation component is neglected, which limits the possibilities for improvements and changes before the model enters an expiration process.

The aim is therefore a theoretical approach in relation to the subject and a discussion of key aspects to consider today, considering the increasing height of the processes of self-assessment and accreditation in educational institutions of higher education.

Key words: instructional design, distance education, educational assessment, educational assessment models, educational materials. 


\section{INTRODUCCIÓN}

En los modelos educativos a distancia, la elaboración de los materiales a utilizar en el proceso de aprendizaje de los estudiantes es una tarea imprescindible, pero además, debe realizarse de manera anticipada a la oferta educativa $y$, también, contemplar un proceso previo de planificación de aquellos contenidos, actividades y objetivos que serán atendidos con los diversos materiales y recursos didácticos (García, 2001a). En ese sentido, el diseño instruccional, en esta modalidad educativa, cobra vital importancia; debido a que, permite la articulación de todos aquellos requerimientos, de orden curricular y didáctico, que deben ser considerados en la elaboración de los materiales y en la oferta misma de asignaturas y cursos (Reigeluth ,1983).

Según Driscoll (2005), el diseño instruccional es en sí mismo un proceso sistemático, mediante el cual se analizan demandas y necesidades, tanto de los estudiantes como de los requerimientos del contexto, a partir de las cuales se establecen objetivos de aprendizaje. Pero además, permite, en el modelo de educación a distancia, la evaluación de las metas propuestas y la mejora de los materiales educativos, y del proceso educativo en sí mismo. De allí la importancia que tiene para las instituciones educativas el contar con un modelo de diseño instruccional, pero, más aún, someterlo a evaluación para su mejoramiento.

Durante los años 2011 y 2012 se lleva a cabo una investigación de carácter evaluativo, cuyo objetivo principal fue, precisamente, realizar una evaluación del modelo de diseño de curso y asignaturas que se venía utilizando en la Universidad Estatal a Distancia de Costa Rica (UNED). Acorde con los lineamientos establecidos en la normativa institucional, existe un proceso de carácter integral que inicia con el diseño de cursos y asignaturas, continúa con la producción de los materiales y la disposición de medios y recursos a utilizar didácticamente, sigue posteriormente con su implementación, para finalizar con la evaluación de los cursos, materiales y medios previamente establecidos.

El interés del estudio surge, a raíz de las diversas discusiones académicas que se realizaron en la
UNED, producto de los retrasos en la producción de los materiales. El tema fue analizado puntualmente en el año 2008, mediante el desarrollo de talleres con la participación de encargados de cátedra, asesores académicos, productores académicos y directores de unidades académicas. En dichos eventos se concluye, por parte de los productores, que el retraso en la producción de materiales, en especial el material didáctico impreso, es producto de la falta de especificación del modelo utilizado para el diseño de los cursos y asignaturas, opinión con la cual, los asesores curriculares participantes, se encontraban en total desacuerdo. A su vez, manifestaron que la problemática estaba ligada más bien, a la no utilización del diseño en los procesos de producción de materiales. Finalmente, los encargados de cátedra señalaron razones varias, entre las que se destaca la necesidad de generar equipos de trabajo que inicien desde la elaboración del diseño del curso o la asignatura y finalicen hasta que se evalúe el material producido (Castillo, 2008).

En general, en ese momento no se encontraron soluciones ante la problemática de los retrasos en la producción de materiales, razón por la cual, se definió realizar una investigación que lograra determinar, de forma mucho más puntual, los factores o situaciones que impiden el cumplimiento de tiempos y cronogramas en la producción. En el proceso previo al inicio del estudio, se llevó a cabo una investigación bibliográfica; cuyo objetivo era indagar el tratamiento del tema de la evaluación de los modelos de diseño, específicamente en la educación superior y bajo la modalidad a distancia, y se concluyó que el interés en él es bastante reciente. Sin embargo, en este trabajo se determina que el diseño instruccional es incluso considerado como una metodología que permite la organización de los diversos procesos de aprendizaje y modelos de enseñanza en el marco de la educación a distancia. Es por ello que interesa, en este artículo, un acercamiento al tema y el análisis de algunos aspectos fundamentales que deberían ser considerados en la actualidad, sobre todo por la atención que han puesto las instituciones de educación superior en la autoevaluación y acreditación de programas, carreras y en las instituciones mismas. 


\section{METODOLOGÍA}

Para la realización de la reseña, se llevó a cabo un proceso metodológico que cumplió con diferentes etapas. En primer lugar, se realizó una investigación de posibles libros relacionados con el tema, que pudiesen ser considerados en el estudio. En esta etapa, los libros fueron seleccionados tomando como criterio principal, los expertos que los elaboraban; se privilegiaron autores a los cuales se les atribuye la experticia en el tema. Para hacer un proceso más selectivo y actualizado, fueron consultados materiales cuyo periodo de vigencia estuviese en un rango de cinco años desde su publicación. Sin embargo, el criterio de antigüedad fue valorado en algunos casos, puesto que los autores que se han destacado por el tratamiento del tema cuentan con publicaciones entre los años sesenta y noventa.

En una segunda fase, se realizó una búsqueda, a profundidad, de experiencias documentadas científicamente sobre evaluaciones de modelos de diseño; que hubiesen sido descritas en reportes de investigación, en un periodo no mayor a los cinco años desde su publicación. Para tal efecto, se utilizaron las bases de datos ERIC y WILSON, ampliamente reconocidas a nivel mundial.

En la tercera fase, se llevó a cabo la búsqueda en revistas especializadas en temas de educación a distancia, como la Revista Interamericana de Educación a Distancia (RIED), la Revista Perfiles Educativos, Redalyc, entre otras, con una antigüedad no mayor a cinco años.

Es importante destacar que, si bien es cierto, el criterio para la selección de las fuentes, en un primer momento, estuvo supeditado a la vigencia de los documentos por asuntos de temporalidad; se consideraron en la investigación fuentes de publicación más antigua, pero que, por su contenido e importancia temática para el trabajo, seguían siendo vigentes.

Posteriormente, la cuarta fase fue la valoración, nuevamente, del material preseleccionado, para lo cual se consideró su pertinencia en el tratamiento del tema y los posibles aportes para el objetivo de la investigación que se realizaría posteriormente. Finalmente, aquellos textos seleccionados fueron sistematizados en fichas de trabajo, que contenían la siguiente información: a) datos generales de la obra o el artículo, b) ideas principales del documento, c) principales aportes del autor y (d) sobre la temática.

\section{RESULTADOS}

\section{Educación a distancia y modelos de diseño}

Para efectos de este trabajo, se define la educación a distancia como una modalidad de aprendizaje, la cual se caracteriza por contemplar la separación física y temporal entre docentes y estudiantes, razón por la cual se utilizan diversos medios y recursos. Así como la producción de materiales educativos, necesarios para el desarrollo de los procesos de aprendizaje (Umaña, 2012). Por otra parte, el diseño instruccional es entendido como un método que permite al docente la organización del proceso educativo, contemplando los objetivos que se persiguen, los contenidos y las actividades requeridas; así como los medios, recursos y materiales educativos que serán utilizados para solventar las situaciones propias que impone el modelo de educación a distancia (Faryaid, 2007). Todo diseño instruccional está sustentado en una teoría educativa determinada, la cual orienta la forma particular en que son entendidos: a) los roles del estudiante y el docente, b) la manera en que se plantean los objetivos y el nivel que se espera lograr, c) las actividades que realizarán los estudiantes como parte de la estrategia de aprendizaje, d) los medios, recursos y materiales educativos que serán requeridos y e) las estrategias de evaluación que serán implementadas (Dick et al., 2005).

\section{- Problemas en la implementación de modelos de diseño instruccional}

La disponibilidad de modelos de diseño es sumamente amplia, dado que existe una tendencia a que cada institución cree el que requiera de acuerdo con los requisitos y necesidades que debe 
solventar (Gustafson \& Branch, 2002). Al respecto, Januszewski y Pearson (1992) encontraron que en la implementación de los modelos, se enfrentan una serie de problemas que deben ser resueltos, ya que es sumamente complejo el proceso en sí del diseño y la elaboración de materiales didácticos (Prasenjit y Shankar, 2009).

Entre los principales problemas que se han encontrado, a la hora de la ejecución de un modelo, está el exceso de tiempo que toma la producción de los materiales instruccionales; problema que no es novedoso (Jones y Richey, 2000). Razón por la cual se ha dado la tendencia de utilizar un modelo al cual se le ha llamado "prototipo rápido", el cual facilita el desarrollo de acciones propias de la capacitación corporativa y programas de computadoras (Wilson, Jonassen \& Cole, 1993). Así, se intenta romper con la estructura de los modelos de diseño instruccional convencionales, ya que permite el desarrollo temprano del producto final (Gravemeijer, 1999). Sin embargo, Tripp y Bichelmeyer (1990) indican que este modelo es de alto costo, por lo que su utilización se ha dado más en empresas corporativas que en instituciones educativas.

En el campo educativo, existe una tendencia a revisar los modelos ante los problemas en su implementación, o bien por las necesidades de cambio que se presentan en las instituciones (Richey \& Klein, 2007). Un caso concreto es el trabajo realizado por la Universidad Nacional de Educación a Distancia de España, quien revisa y adecua su modelo de diseño instruccional de manera constante (García, 2001b). También, se encontró que otras universidades a distancia se han interesado en evaluar sus modelos, con el propósito de realizar mejoras en la oferta educativa, tal es el caso de aquellas de origen mexicano (Gonzáles \& Hernández, 2006). De igual forma, se destaca el caso de la Universidad de Oberta - Cataluña en España, donde el tema del análisis del diseño y los procesos de producción de materiales está bajo constante observación y estudio. (Barberá, comunicación personal, abril, 2010).

Monedero (2009) menciona, en concordancia con el tema, que en la actualidad se tiene el reto de la revisión de los modelos de diseño; en particular, si se considera que las políticas educativas, y sobre todo los contextos sociales, apuntan hacia la búsqueda de espacios de convergencia entre las instituciones educativas; de manera que se promueva la movilización de los estudiantes en las universidades.

Con relación al tema de las revisiones de los modelos de diseño, Durdu, Yalabik y Cagiltay (2009) mencionan que, las mismas deben efectuarse en periodos cortos, de manera que con prontitud se incorporen los cambios necesarios para el mejoramiento de su efectividad.

En términos generales, Tracey y Richey (2007) encontraron que, entre las principales dificultades al implementar los modelos de diseño, están: a)la definición de objetivos, b) el grado de especificación de los contenidos a desarrollar, c) el detalle de las actividades de aprendizaje, d) la selección y especificación de los medios y materiales educativos que se requieren para el desarrollo de las actividades de aprendizaje, y d) la definición de estrategias y actividades para la evaluación de los aprendizajes.

Sobre las dificultades encontradas en lo relativo a la definición de los objetivos, se encontró que pueden repercutir en la propuesta educativa y en la producción de materiales, ya que si son muy generales, o bien, sumamente específicos, dificulta su contextualización y la definición del tipo de material (Willey y Edwards, 2002). Para Wilson (1997), la popularización del uso de taxonomías está ligada a la necesidad de generar procesos más ágiles y que permitan claridad en los logros y metas de aprendizaje esperados.

Sobre el tratamiento de los contenidos, Díaz (2010) indica que, en la Universidad Nacional Abierta de Venezuela, ha sido necesaria la revisión de las especificaciones de estos, dado que se han enfrentado retrasos en la producción de los materiales educativos por la falta de claridad de estos. Igual situación se presenta en la especificación del diseño de las actividades de aprendizaje (Mena, 1992), en las cuales, la poca claridad y definición retrasa los procesos de diseño de cursos y asignaturas, al mismo tiempo que la producción de materiales y finalmente la selección de los medios, según las actividades que se desea que el estudiantado realice. (Januszewski \& Molenda, 2008). 
Algunas soluciones, relacionadas con el proceso de selección de medios y la producción de materiales, están en la posible reutilización de material preexistente, lo cual disminuye costos y tiempos (Zimmas, Kleftourios y Valkano, 2009). Además, según lo proponen Lara (2009) y Pensa (2000), esta medida deja de lado la dependencia del material impreso y facilita la modificación de los recursos.

En síntesis, la evaluación de los modelos de diseño permite, de forma oportuna, enfrentar los cambios y las adaptaciones que se requieren para que los mismos cumplan con las exigencias, tanto de las instituciones como del contexto. Por tanto, todo proceso de evaluación debe tener como premisa el establecimiento de la relación comparativa, entre los objetivos que dieron origen al modelo y su puesta en práctica; para que los resultados favorezcan la toma de decisiones oportuna para la mejora del mismo (Stufflebeam \& Shinkfield, 1989).

\section{- Evaluación educativa}

Se entiende por evaluación educativa, aquel proceso de carácter científico que, se asume, será realizado en un ambiente educativo controlado (Pons, 1991). A lo largo de la historia se pueden encontrar importantes esfuerzos en este campo, entre los que destacan aquellos propuestos por Tyler (1949), quien fue pionero en el área, donde se caracterizó por desarrollar un modelo curricular, en el cual, uno de sus componentes era la evaluación de dicho modelo y así, determinar el grado de cumplimiento de los objetivos educativos (Pons, 1991). También se destaca el trabajo de Cronbach (1963), quien toma el modelo tyleriano como punto de partida y, en una reelaboración del mismo, plantea que los resultados de la evaluación deben proveer información para la mejora del proceso de la enseñanza (Pons, 1991). Sin embargo, estos esfuerzos fueron incipientes hasta la década de los años setenta, cuando nuevamente el tema de la evaluación empieza a cobrar importancia, no solo para control, sino para la toma de decisiones y mejora de los programas educativos.

Según Scriven (1967), para el logro de los objetivos educativos, debe considerarse siempre la evaluación como parte del proceso. Desde su perspectiva, la evaluación necesariamente tendría que ser formativa y sumativa. La evaluación formativa permitiría la realización de cambios y mejoras durante el proceso, mientras que la sumativa se realizaría al finalizar los objetivos, siendo orientada hacia una evaluación propiamente de los productos obtenidos.

Stake (1975) retoma la propuesta de Scriven y propone el modelo de la evaluación respondiente; de acuerdo con su modelo, quien evalúa debe tomar en cuenta el criterio de todas las personas involucradas, con lo cual se espera que se encuentren posibles propuestas de mejora ante las dificultades que surjan en el proceso. Desde este modelo, el criterio de profesores y estudiantes es sumamente importante, por lo que se sugiere la utilización de metodologías que permitan el rescate de las opiniones de todos los involucrados.

Finalmente, para los efectos de este trabajo, se retoma el modelo de evaluación de programas, desarrollado por Stufflebeam y Shinkfield (1989), el cual parte de la premisa de que toda evaluación debe proveer datos precisos que permitan tomar las decisiones necesarias y en el momento en que se requiera, en pro del mejoramiento del programa. Para estos autores, sea cual sea la naturaleza del programa o modelo, pero enmarcado en el contexto social o educativo, es susceptible de mejora; es por ello que el principal interés de este modelo es el suministro de la información necesaria para el perfeccionamiento del mismo, de manera permanente.

El modelo de Stufflebeam y Shinkfield (1989) consta de cuatro procesos: contexto, entrada o insumo, proceso y, producto o resultado (CIPP, por sus siglas en inglés). En el primero de ellos, se lleva a cabo la evaluación de contexto, donde el propósito principal es la caracterización del ambiente en que se inscribe la institución o programa educativo; con ello se pretende potenciar las ventajas encontradas en él y, a su vez, disminuir las limitantes.

El segundo proceso en el modelo CIPP es la evaluación de insumos, en el cual se tiene como objetivo prevenir posibles dificultades, para ello se debe elaborar un plan de manejo de los recursos con que se cuenta. La evaluación de proceso es la tercera etapa, 
en este caso el interés se enfoca en valorar el programa en su etapa de implementación. Por lo tanto, se espera que los participantes cuenten con constante realimentación, de manera que se puedan implementar cambios inmediatos para la obtención de mejores resultados.

El último proceso se denomina evaluación de producto, cuya intención es, precisamente, evaluar los logros alcanzados según los objetivos propuestos; es por ello que se realiza cuando el programa ha finalizado.

Posterior a la propuesta de Stufflebeam y Shinkfield (1989), se encontró que existe una tendencia a utilizar algunos de los procesos planteados por los autores para la evaluación de programas y modelos, tal como lo propone Chiape (2008).

\section{- La evaluación de los modelos de diseño}

Según indican Bautista, Martínez y Sainz (2001), la evaluación del diseño es ineludible, puesto que permite la realización de cambios necesarios en el mismo y, por ende, se contribuye al logro de su finalidad última, que es la producción de los materiales. Sin embargo, hay poca referencia de prácticas evaluativas en modelos de diseño, principalmente porque los mismos se valoran mediante su uso (Richey y Klein, 2007). A pesar de ello, se hallan indicios de que en los últimos años hay avances, en lo relativo a la intencionalidad de implementar metodologías evaluativas para los modelos de diseño (Tracey y Richey, 2007).

Según Richey (2005), se pueden distinguir básicamente cinco enfoques para la validación de los modelos de diseño: la revisión de expertos, la documentación de usabilidad, la investigación de los componentes del diseño, la evaluación del campo y el desarrollo de pruebas controladas.

El enfoque orientado a la revisión de expertos se centra en la validez del diseño, para lo cual recurre, como su nombre lo indica, a la revisión de especialistas, quienes emiten su criterio sobre las ventajas y desventajas de utilizar el modelo. Básicamente, la validez será sustentada según las calidades de los participantes y la cantidad de personas consultadas (Tracey \& Richey, 2007).

En el caso del enfoque orientado hacia la validación de documentos de usabilidad, se pretende el registro sistemático de todas aquellas posibles situaciones que se presenten mientras el diseño se implementa, por lo que, la organización de la información y su análisis con regularidad, son fundamentales (Richey, 2005).

El tercer enfoque es el de la investigación de los componentes del diseño, donde el interés se concentra en la validez interna del modelo de diseño, es por ello que se enfoca en el estudio de los procedimientos (Tracey \& Richey, 2007). Previo al estudio, deben ser definidos los diversos procesos o etapas del diseño y, finalmente, se puede optar por la evaluación de uno o todos los componentes del modelo.

El cuarto enfoque de validación de modelos es la evaluación de campo, el cual consiste en la puesta en práctica de acciones para evaluar el modelo mientras se implementa. Por último, se tiene el enfoque orientado hacia la aplicación de pruebas controladas; su interés central es la validación de modelos mediante la efectividad de los mismos, donde las pruebas estandarizadas se convierten en instrumentos fundamentales.

A partir de los resultados encontrados en este ámbito, queda en evidencia que, además de la necesidad de evaluar los modelos, existe otra decisión importante relacionada con el enfoque que se le dé a la misma; puesto que, tal como se ha podido identificar, los ámbitos de estudio, la metodología y los instrumentos, así como los participantes, difieren sustantivamente, según el interés que oriente la evaluación.

\section{CONCLUSIONES}

La revisión de literatura efectuada deja claro que el estudio de los modelos de diseño instruccional es en la actualidad pertinente y debería ser parte de las líneas de investigación de las instituciones educativas con un modelo de educación a distancia. 
Producto de la investigación realizada, se concluye que existe una gama bastante amplia de modelos de diseño instruccional, los cuales son producto de las necesidades de las instituciones, por tanto no se puede establecer una normalización de la forma en que estos se construyen. A pesar de ello, es de esperar que las propuestas surjan sustentadas teóricamente, así queda claro que no son iniciativas activistas y descontextualizadas.

En relación con el tema específico de la evaluación educativa, se encuentra que, a pesar de estar aún en un proceso de crecimiento, es sumamente necesario; lo cual se demuestra en la tendencia de las instituciones educativas a participar en procesos de mejora continua y en busca de la acreditación. Al respecto, en la UNED debería establecerse, al menos, algunas líneas de estudio al respecto, con las cuales se puedan generar conocimientos que permitan, a corto plazo, el mejoramiento de su modelo de diseño de curso.

La evaluación de los modelos de diseño instruccional está poco sistematizada, lo cual es parte de una práctica habitual, en donde los modelos son validados en su ejecución. Sin embargo, este tipo de acciones no permite el análisis y la toma de decisiones para la mejora, a partir de datos puntuales.

Finalmente, la evaluación de los modelos de diseño instruccional debe estar sustentada a partir del enfoque desde el cual interesa validarlo, por tanto, implica previamente la toma de decisiones sobre las principales necesidades que intenta solventar. Ante esta situación, es evidente que la metodología utilizada para la evaluación de un modelo, depende de las particularidades del mismo.

\section{REFERENCIAS}

Castillo, T. (2008). Recorrido histórico acerca de la calidad $y$ producción de los materiales didácticos: Problemas identificados. Costa Rica: Material mimeografiado.

Chiape, A. (2008). Diseño instruccional: Oficio, fase y proceso. Revista Informática Educativa, 11(2), 229-239.
Cronbach, L. (1963). Course improvement through evaluation [Mejoramiento en curso a través de la evaluación]. Teachers College Record, 64, 672-683.

Díaz, R. (2010). Producción de materiales impresos modelo UNA. Recuperado de http://institución.ac.cr/ global/tecnología/impresa/articulos/produciondemateriales.htm

Dick, W., Carey, L. \& Carey, J. (2005). The systematic design of instruction (6ta. ed.). New York, NY: Allyn \& Bacon.

Durdu, P. O., Yalabik, N. \& Cagiltay, K. (2009). A distributed on line curriculum and courseware development model [La distribución del curriculum en línea y el desarrollo de los cursos]. Educational Technology \& Society, 12(1), 230-248.

García, L. (2001b). La innovación permanente en la UNED: del material impreso a la tecnología UMTS. Edutec, Revista Electrónica de Tecnología Educativa, 14. Recuperado de http://edutec.rediris.es/Revelec2/ Revelec14/aretio.html

Gravemeijer, K. (1999). How emergent models may foster the constitution of formal mathematics [Cómo los modelos emergentes pueden fomenter la conformación de la matemática formal]. Mathematical Thinking and Learning, 1(2), 155-177.

Gustafson, K. \& Branch, R. (2002). Survey of instructional development models [Estudio del desarrollo de modelos instruccionales] (4a. ed.). (No. de servicio de reproducción de documento ERIC ED477517). Recuperado de la base de datos ERIC.

Faryaid, Q. (2007). Instructional design models. What a Revolution [Modelos de diseño instruccional. Qué revolución?]. (No. de servicio de reproducción de documento ERIC ED495711). Recuperado de la base de datos ERIC.

Januszewski, A. \& Pearson, R. (1992). Problem identification techniques: So what's the problem? [Técnicas de identificación de problemas ¿cuál es el problema?]. (No. de servicio de reproducción de documento ERIC ED347996). Recuperado de la base de datos ERIC.

Jones, T. \& Rickey, R. (2000). Rapid prototyping methodology in action: a development study [Prototipo rápido metodológico en acción: un estudio de su desarrollo]. Educational Technology Researche Development, 48(2), 63-80. 
Lara, C. (2009). Propuesta para la elaboración de guías didácticas en programas a distancia. Recuperado de http://eduvirtual.chapingo.mx/archivos/guia_didactica .doc

Mena, M. (1992). Nuevos enfoques pedagógicos para mejorar la producción de materiales en la educación a distancia. Journal of distancie educational, 7(3), $121-130$

Monedero, J. (2009). El diseño de los materiales educativos ante un nuevo reto en la enseñanza universitaria: el espacio europeo de educación superior. Revista Interuniversitaria de Formación de Profesorado, 21(1), 51-68.

Pensa, D. (2000, junio). Producción de materiales educativos: Del texto al hipertexto. Documento presentado en Virtual Educa en Madrid, España.

Prasenjit, Ch. Vijay, M. \& Shankar, Ch. (2009). Selection of materials using compromise ranking and outranking methods [Selección de los materiales utilizados según el compromiso y metodos de superación ]. Materials \& Desing, 30, 4043-4053.

Pons, J. (1991). La evaluación educativa de los medios instruccionales. Revista Enseñanza, 7, 9-17.

Richey, R. \& Klein, J. (2007). Design and development research: Methods, strategies and issues [Diseño y desarrollo de la inviestigación: métodos, estrategias y tareas]. Mahwah, NJ: Lawrence Erlbaum Associates.

Richey, R. (2005). Validating instructional design and development models [Validación y desarrollo de modelos de diseño instruccional]. En J. M. Spector \& D. Wiley (Eds.), Innovations in instructional technology. Mahwah, NJ: Lawrence Erlbaum.

Stufflebeam, D. \& Shinkfield, A. (1989). Evaluación sistemática: Guía teórica y práctica. Barcelona, España: Piados.

Scriven, M. (1991). Beyond formative and summative evaluation [Más allá de la evaluación formativa y sumativa]. En M. W. McLaughlin \& D. C. Phillips (Eds.). Evaluation and education: At quarter century (pp.19-64). Chicago, IL: University of Chicago Press.
Stake, R. (1975). Program evaluation: Particulary response [Evaluación de programa: Respuesta particular]. En Occasional Paper, 5. Universidad de Western Michigan.

Tracey, M. W. \& Richey, R. C. (2007). ID model construction and validation: A multiple intelligences case [Construcción y validación de un modelo de diseño instruccional: un caso de inteligencias múltiples]. Educational Technology Research and Development, 55(4), 369-390.

Tracey, M. (2007). Design and development research: A model validation case [Diseño y desarrollo de la investigación: un caso de validación de modelo]. Educational Technology Research and Development, $57,553-571$.

Tyler, R. (1949). Basic principles of curriculum and instruction ([Principios básicos de curriculum e instrucción]. Chicago, IL: University of Chicago Press.

Universidad Estatal a Distancia. (2010). Reglamento de gestión académica de la Universidad Estatal a Distancia (2da. ed.). San José, Costa Rica: Autor.

Wiley, D. \& Edwards, E. (2002). Online self-organizing social systems: The descentralizad future of online learning [Auto organización de los sistemas sociales. El futuro descentralizado del aprendizaje en línea]. The Quartely Review of Distance Education, 3(1), 33-46.

Wilson, B. G., Jonassen, D. H. \& Cole, P. (1993). Cognitive approaches to instructional design [Los enfoques cognitivos en el diseño instruccional]. En G. M. Piskurich (Ed.), The ASTD handbook of instructional technology (pp. 21.1-21.22). New York, NY: McGraw-Hill.

Zimmas, A., Kleftourios, D. \& Valkanos, N. (2009). IDEL-A simple instructional design tool for e-learning [IDEL-A herramienta simple de diseño instruccional para el aprendizaje en línea]. Proceedings of World Academy of Science, Engineering and Technology, 37, 366-372. 